Annuaire suisse de politique de développement

$14 \mid 1995$

Annuaire Suisse - Tiers Monde 1995

\title{
La Suisse et les nouveaux efforts internationaux pour juguler la corruption dans le monde
}

\section{Mark Pieth}

\section{(2) OpenEdition}

\section{Journals}

Édition électronique

URL : http://journals.openedition.org/aspd/1482

DOI : $10.4000 /$ aspd. 1482

ISSN : 1663-9669

Éditeur

Institut de hautes études internationales et du développement

\section{Édition imprimée}

Date de publication : 1 février 1995

Pagination : 249-254

ISSN : 1660-5934

\section{Référence électronique}

Mark Pieth, "La Suisse et les nouveaux efforts internationaux pour juguler la corruption dans le monde ", Annuaire suisse de politique de développement [En ligne], 14 | 1995, mis en ligne le 04 mai 2013, consulté le 08 septembre 2020. URL : http://journals.openedition.org/aspd/1482 ; DOI : https:// doi.org/10.4000/aspd. 1482 


\title{
La Suisse ef les nouveaux efforts internationaux pour juguler la corruption dans le monde
}

\author{
Mark Pieth
}

\section{Le débat public sur la corruption}

Un problème longtemps passé sous silence fait actuellement la «une» des médias. La corruption, ou les agissements assimilables à la corruption, prennent des dimensions universelles. La "petite corruption" de la vie quotidienne a longtemps nourri les conversations des voyageurs qui revenaient du Tiers Monde. Puis les industriels qui souhaitaient établir des relations d'affaires dans ces régions ont raconté, avec un haussement d'épaules, que même et surtout à grande échelle, il n'était pas question de passer un contrat sans dessous-detable.

En dépit des "affaires" qui secouent de temps à autre le monde industrialisé, il est nouveau que l'on admette l'existence en Europe - et pas seulement en Italie - d'une corruption qui a pris des proportions endémiques.

La corruption en tant que mécanisme intrinsèque d'une économie s'est révélée dans toute son ampleur avec l'ouverture des marchés de l'Est, et plus particulièrement de la Russie. Même si le népotisme des fonctionnaires imprégnait déjà la vie économique sous le régime soviétique, la corruption représente un élément de l'ouverture qui prend une tout autre dimension. Les associations d'idées qu'elle suscite vont du "wild West" au "capitalisme manchestérien".

\section{Délimitation de la notion de corruption}

II va de soi que le débat public - surtout dans les médias - se préoccupe peu de clarté. Corruption est synonyme d'affaires douteuses. Cette notion sert également de code; elle crée de nouveaux boucs émissaires sur qui l'on peut reporter inquiétudes diffuses et craintes de privation. 
La définition de cette problématique relève avant tout du droit pénal. En ce qui concerne la corruption de fonctionnaires, le droit prévoit la corruption proprement dite (active et passive) qui consiste à offrir une prestation à un fonctionnaire contre un service auquel on n'a pas droit (1). Cet pratique peut léser des tiers; elle porte de toute manière atteinte à la confiance en l'intégrité de la fonction publique. La législation pénale prévoit en outre, d'une manière générale, la variante du simple cadeau considéré comme suffisant pour compromettre la confiance des administrés dans la fonction publique (2). De leur côté, les lois qui visent à réprimer la concurrence déloyale comprennent également des dispositions pénales contre la corruption de particuliers à l'insu de leur employeur (3). Ce comportement tombe en partie sous le coup du délit classique de la gestion déloyale.

Réduire le phénomène à sa dimension pénale ne permettrait guère d'en examiner les divers aspects: le droit pénal se concentre par définition sur les responsabilités individuelles et n'est guère en mesure de dépasser le délit concret pour s'intéresser aux structures persistantes et aux réseaux qui font de la corruption un moteur des activités économiques et sociales. Et c'est pourquoi la législation pénale est dépassée lorsqu'elle doit affronter la dimension internationale de la "grande corruption".

Mais ce n'est pas un hasard si le débat public sur la corruption s'est limité jusqu'à présent à l'aspect pénal dans notre pays. Sans doute est-il important pour l'identité helvétique d'apprendre qu'un Raphael Huber a pu longtemps exiger et recevoir des pots-de-vin, que le système des autorisations de complaisance dans la restauration a pu fonctionner durablement. II s'agit toujours d'une série de cas isolés, tout au moins si nous limitons notre examen à la corruption proprement dite. Cette discussion occulte le fait que la corruption ne représente que l'aspect partiel d'un thème plus vaste, qui est le patronage et le clientélisme (4). Alors que la corruption prend la forme d'avantages pécuniaires - tout en recherchant d'ailleurs des relations durables - les comptes se règlent au coup par coup dans la monnaie du pays considéré. Le trafic d'influence est en revanche une affaire à long terme: les faveurs accordées dans le cadre d'un réseau de relations se rendent parfois des dizaines d'années plus tard (les parlementaires suisses en savent quelque chose).

L'absence, en Suisse, de loi sur le financement des partis politiques montre à quel point la criminalisation du clientélisme peut être sélective. Une bonne part de la "corruption" dont souffrent nos pays voisins se rapporte au financement illicite des partis. Le fait même de renoncer volontairement à exiger la transparence financière des activités politiques (donc de légaliser des arrangements secrets) est le moyen d'éviter - à tort ou à raison - une forme possible de délinquance. La non-criminalisation agit comme une mesure préventive, ce qui prouve que la criminalité peut être affaire d'inculpation.

Le fait de restreindre la notion de corruption à l'acception qu'en donne le code pénal suisse voile en bonne partie sa dimension internationale: les délits répertoriés dans notre code pénal (en particulier les art. 288,315 et 316 CPS) ont pour objet de sauvegarder la confiance du peuple envers ses propres 
institutions. Ces normes juridiques sont issues de la genèse de l'Etat national. Et l'obstacle de la "pénalité réciproque" peut être éventuellement surmonté par le fait que pratiquement toutes les nations connaissent des règles de droit analogues. Cependant, corrompre des fonctionnaires d'un Etat étranger n'est pas un acte condamnable aux termes du code pénal suisse. De plus, la jurisprudence (5) appliquée jusqu'à présent en Suisse comme dans d'autres pays considère - tout au moins en principe - la corruption internationale comme frais d'acquisition fiscalement déductibles, et favorise ainsi indirectement la corruption.

\section{La corruption internationale et la Suisse}

Même en donnant un sens étroitement pénal à la notion de corruption, il est douteux que l'on puisse faire abstraction de l'aspect international du phénomène. La Suisse est triplement touchée par la corruption internationale:

En tant que pays exportateur, la Suisse participe activement - comme d'autres Etats industrialisés - à la corruption de fonctionnaires étrangers, du Tiers Monde en particulier. Même si certaines entreprises respectent les principes de la Chambre de commerce internationale (CCl) (6) et si d'autres combattent la corruption à l'étranger tout au moins dans leur réglementation interne, beaucoup d'exportateurs se voient contraints de verser des dessous-de-table sans contrepartie. Des entreprises suisses freinent ainsi - le plus souvent malgré elles - le processus de développement des pays du "Sud" et de l'"Est".

Par ailleurs, la Suisse peut aussi être le "lieu du crime": l'affaire Lockheed, par exemple, nous a révélé qu'il est traditionnel d'avoir un compte en Suisse pour pouvoir verser les pots-de-vin à partir de ce pays. Cette impression est confirmée par les méthodes pratiquées en Italie (7).

Songeons enfin au "blanchiment de pots-de-vin" sur territoire suisse: beaucoup de fonctionnaires essaient de dissimuler et de légaliser chez nous les fruits de leur vénalité, avec l'aide occasionnelle de financiers suisses.

\section{Efforts internationaux pour juguler la corruption}

A la suite du scandale Lockheed, qui a révélé au monde entier à quel point non seulement les avionneurs américains mais beaucoup de multinationales corrompaient régulièrement les plus hauts fonctionnaires étrangers, I'ONU a tenté d'élaborer une convention contre la corruption internationale. Ses efforts dans ce sens se sont poursuivis de 1976 à 1979 et ont échoué finalement face aux divergences insurmontables entre nations industrialisées et pays du Tiers Monde d'une part (surtout à propos du jugement à porter sur les versements au régime d'apartheid en Afrique du Sud), ainsi qu'entre Etats industrialisés eux-mêmes (à propos de la conception étasunienne de l'effet extraterritorial du droit pénal) (8). 
D'autres tentatives, de l'OCDE en 1976 (9) et de la Chambre de commerce internationale en 1977 (10), n'ont pas eu d'incidences profondes sur les pratiques de la corruption internationale.

Les Etats-Unis ont relancé en 1989, dans le cadre de l'OCDE, l'idée de passer un accord contre "les paiements illicites dans les affaires internationales". Les pays membres de l'OCDE n'ont pas suivi cette proposition, mais se sont entendus pour élaborer une recommandation qui a été adoptée le 27 mai 1994 par le Conseil de l'OCDE, et entérinée depuis lors par les ministères compétents des Etats membres.

L'importance de ce texte tient au fait que les pays industrialisés s'y déclarent prêts à tout entreprendre pour enrayer de leur côté la corruption active. Ils ont adopté à cet effet une "procédure de suivi" qui évaluera régulièrement les progrès de la mise en oeuvre et élaborera des propositions en vue de préciser la teneur de cette recommandation. Au départ, il s'agit avant tout de briser toute une série de tabous internationaux que l'on se gardait jusqu'ici d'aborder de front, en particulier le traitement fiscal des pots-de-vin et la criminalisation de la corruption de fonctionnaires étrangers.

Il va de soi que des recommandations non impératives ( soft law) ne peuvent être efficaces que si leur application fait l'objet d'une pression réciproque (peer pressure).

Alors que la Banque mondiale lie régulièrement l'octroi de ses crédits à des conditions de ubonne gestion des affaires publiques" (good governance) portant sur la démocratisation ainsi que sur des réformes administratives et économiques (11), le monde industrialisé doit pour sa part manifester par cette directive son intention d'intervenir contre la corruption active. Mais il faut bien se rendre compte, en l'occurrence, que le mobile principal des nations exportatrices était leur intérêt de diminuer les distorsions de la concurrence.

Une bonne part des règles de "bonne gestion des affaires publiques" consiste à supprimer les restrictions à l'importation, donc le pouvoir plus ou moins discrétionnaire de certains fonctionnaires. Cela réduit les possibilités de corruption, tout en favorisant l'intérêt des pays industrialisés à voir s'ouvrir les marchés du "Sud". Cependant, aussi bien les règles de l'OCDE que celles de la good governance ne signifient en aucune manière qu'une simple dérégulation puisse suffire à juguler la corruption. II faut au contraire créer des conditions de transparence par le biais de normes administratives bien précises, par exemple dans les domaines de la comptabilité ou de la législation sur les cartels. L'application de ce principe doit être particulièrement rigoureuse face à des protagonistes qui peuvent se passer des règles de la concurrence légale et disposent de "caisses noires" bien garnies provenant de l'économie souterraine. La dérégulation est d'autant moins une panacée lorsque les capitaux du crime organisé (et en particulier du marché de la drogue) affluent dans le circuit économique légal.

L'initiative de l'OCDE a été reprise depuis lors par diverses organisations telles que la Conférence européenne des ministres de la justice à Malte et le Conseil de l'Europe, qui la poursuit dans son propre groupe de travail. 


\section{Mesures contre la corruption prises par la Suisse}

Nous avons déjà parlé des délits traditionnellement répertoriés par notre code pénal en matière de corruption active et passive, délits qui se limitent à la corruption de fonctionnaires suisses.

L'arsenal répressif s'est élargi avec l'entrée en vigueur de la loi contre le crime organisé du 1er août 1994; on sait en effet que la corruption et le chantage comptent parmi les instruments de travail favoris des organisations criminelles. Un aspect de cette loi particulièrement important pour notre pays est qu'il suffit désormais de soutenir une telle organisation dans ses activités criminelles pour être punissable, même sans la preuve que l'on a trempé dans la préparation ou l'accomplissement d'actes bien précis (12).

De plus, les nouvelles dispositions du droit de confiscation permettent de résorber plus facilement les capitaux acquis par des méthodes illicites. En particulier les fonds en possession du crime organisé peuvent être confisqués en partie ou en entier (13). Celui qui est accusé d'avoir soutenu une organisation criminelle devra à l'avenir prouver, en plus, que l'organisation incriminée n'a pas la haute main sur son patrimoine s'il ne veut pas que celui-ci soit entièrement confisqué (inversion de la charge de la preuve) (14).

On connaît maintenant les effets de la nouvelle disposition pénale sur le blanchiment d'argent sale en relation avec la corruption de fonctionnaires suisses (art. 305 bis CPS): la corruption passive est un délit qui a donc valeur d'antécédent du blanchiment. Mais une question qui n'a pas encore été réglée est de savoir si la corruption de fonctionnaires étrangers est assimilable à un antécédent au sens des nouvelles dispositions sur le blanchiment d'argent sale (15). En tout état de cause, la Commission fédérale des banques considère actuellement que le fait d'accepter intentionnellement les pots-de-vin versés à des fonctionnaires suisses ou étrangers constitue une violation des normes imposées par la surveillance des banques (16).

Toute une série de règles relevant du droit civil et administratif ont indirectement pour effet de prévenir la corruption. Il y a par exemple les prescriptions relatives à la comptabilité, les dispositions visant à limiter le droit d'accorder des autorisations ou à passer des commandes, ainsi que les directives de contrôle qui s'y rapportent. D'autres dispositions contribuant à éviter la corruption au sens large du terme sont les restrictions d'éligibilité à des fonctions publiques en vue de combattre le népotisme, ainsi que les règles de récusation correspondantes.

\section{L'accueil en Suisse des propositions internationales}

Une bonne partie des thèmes abordés ici font déjà l'objet de débats au sein du Parlement helvétique et de ses commissions. C'est ainsi que la Commission de l'économie et des redevances (CER) des Chambres fédérales examine actuellement les suites à donner à l'initiative Carobbio sur la non-recon- 
naissance des déductions fiscales portant sur des pots-de-vin (17). D'autres interventions visent à étendre le caractère pénal de la corruption active aux fonctionnaires étrangers (18).

Le Conseil fédéral, de son côté, a pris connaissance avec approbation des recommandations formulées par l'OCDE, et mis sur pied un groupe de travail interdépartemental chargé d'en étudier la mise en application. Les représentants du monde économique se montrent dans l'ensemble très favorables à des interventions efficaces pour juguler la corruption. Mais ils manifestent encore une certaine réserve envers le processus engagé par l'OCDE. La réussite des mesure concrètes dépendra de la coopération internationale.

\section{Notes}

1 Cf. art. 288, 315 CPS

2 Exemple pour la Suisse: art. 316 CPS

3 Exemple pour la Suisse: art. 4 a et b LCD

4 Cf. Schmuel N. Eisenstadt/Louis Roninger, Patrons, Clients and Friends, Cambridge 1984, et Gioia Weber Pazmino, Klientelismus, Zurich 1991.

5 Cf. circulaire de l'Administration fédérale des contributions du 8.11.1946.

6 Extorsion and Bribery in Business Transactions, Report adopted by the 131st. Session of the Council of the ICC, 29 novembre 1977.

7 Cf. articles sur la "pista Svizzera” des affaires de corruption dans le Nord de l'Italie: p.ex. Tages-Anzeiger du 22.9.1993, ou rapport annuel 1993 de la Commission fédérale des banques, p. $32 \mathrm{~s}$.

8 Cf. Foreign Corrupt Practices Act du 1977, modifié par l'Omnibus Trade and Competitiveness Act 1988.

9 Directive OCDE pour les multinationales du 21 juin 1976.

10 Cf. note 6.

11 La Banque mondiale a élaboré en 1993, en relation avec l'octroi d'un crédit au Kenya, une "Economic Governance: Anticorruption Policy Matrix" indiquant les problèmes que le gouvernement kenyan est tenu de traiter.

$12 \mathrm{Cf}$. art. 260 ter, en vigueur depuis le 1er août 1994, message du Conseil fédéral, FF 1993 III 277 ss

13 Art. 59 ch. 3 CPS

14 Id. 2 phrase.

15 Cf. arrêt non publié de la chambre d'appel tessinoise du 4.9.1992, p. 71, ainsi que Niklaus Schmid, Anwendungsfragen der Straftatbestände gegen die Geldwäscherei, vor allem StGB Art. 305 bis, in: Geldwäscherei und Sorgfaltspflicht, Schweizerischer Anwaltsverband $1991 \mathrm{p}$. 111 ss., et Paolo Bernasconi, Die Bestechung von ausländischen Beamten nach schweizerischem Straf- und Rechtshilferecht zwischen EG-Recht und neuen AntikorruptionStaatsvertrăgen, in ZStrR 109 (1992) p. 383 ss.

16 Rapport annuel de la CFB 1993, p. 32.

17 Initiative parlementaire Carrobio du 16 juin 1993 (93.440).

18 Question ordinaire urgente Rechsteiner du 31 mai 1994 (94.1059). 